PROCEEDINGS OF THE AMERICAN MATHEMATICAL SOCIETY

Volume 135, Number 4, April 2007, Pages 1159-1162

S 0002-9939(06)08701-6

Article electronically published on October 11, 2006

\title{
A NEW PROOF OF PROXIMINALITY FOR $M$-IDEALS
}

\author{
V. INDUMATHI AND S. LALITHAMBIGAI
}

(Communicated by Jonathan M. Borwein)

\begin{abstract}
We give a new and a simple proof of proximinality for $M$-ideals. Unlike the known proofs, our proof derives proximinality of $M$-ideals directly from the definition of an $M$-ideal, using the Bishop-Phelps theorem.
\end{abstract}

More than one proof is available for proximinality of $M$-ideals. We refer the reader to the Notes and Remarks following Chapter II in [5], for details. The earlier proofs of Alfsen and Effros [1] (using "dominated extensions") and that of Ando [2] (using the notion of "splittable convex sets"), are elaborate and technically involved. A simpler proof was given in Proposition 6.5 of 3 and Lemma 1.1 of 8 (see also Proposition 1.1 of $[5]$ ), using the fact that $M$-ideals have the 3 -ball and hence the weaker $1 \frac{1}{2}$ - ball intersection property. This property was used to construct a Cauchy minimizing sequence for each element to be approximated. Later, in [6], a geometric property of $M$-ideals was used to do the same.

In this paper we present an easy, short proof of proximinality of $M$-ideals using just the definition of $M$-ideals and the Bishop-Phelps theorem.

We begin with some notation and definitions that are needed in the sequel. We assume that all subspaces are closed. Throughout $X$ denotes a real Banach space and $X^{*}$ denotes the dual of $X$. For any $x$ in $X$ and a subspace $Y$ of $X$, we denote by $d(x, Y)$ the distance of $x$ from $Y$. Let

$$
P_{Y}(x)=\{y \in Y:\|x-y\|=d(x, Y)\} .
$$

If $P_{Y}(x)$ is a non-empty set for each $x$ in $X$, then the subspace $Y$ is said to be proximinal in $X$. For a subspace $Y$ of $X$, let $Y^{\perp}$ denote the annihilator of $Y$ in $X$, that is,

$$
Y^{\perp}=\left\{f \in X^{*}: f \equiv 0 \text { on } Y\right\} .
$$

For $f$ in $X^{*}$, the restriction of $f$ to $Y$ is denoted by $f_{\mid Y}$. The following definitions can be found in [5].

Definition 1 ([5]). A subspace $Y$ of a Banach space $X$ is called an $L$-summand of $X$ if there exists a subspace $Z$ of $X$ such that $X=Y \oplus_{1} Z$, the $l_{1}$-direct sum

Received by the editors November 14, 2005.

2000 Mathematics Subject Classification. Primary 46B20, 41A50, 41A65.

Key words and phrases. Proximinal subspace, $M$-ideal.

The first author was partially supported by DST-NSF project No. DST/INT/US(NSF-RPO0141)/2003. 
of $Y$ and $Z$. This implies that if $x=y+z$ for $x$ in $X, y$ in $Y$ and $z$ in $Z$, then $\|x\|=\|y\|+\|z\|$.

Definition 2 ([5]). A subspace $Y$ of a Banach space $X$ is called an $M$-ideal in $X$ if $Y^{\perp}$ is an L-summand in $X^{*}$.

If $Y$ is an $M$-ideal in $X$, then there exists a subspace $Z$ of $X^{*}$ such that

$$
X^{*}=Y^{\perp} \oplus_{1} Z .
$$

Using the canonical isometric isomorphism $Y^{*} \simeq X^{*} / Y^{\perp} \simeq Z$, one can conclude (see Proposition 1.12 of [5]) that

$$
Z=\left\{f \in X^{*}:\|f\|=\left\|f_{\mid Y}\right\|\right\} .
$$

Remark 1. If $Z$ is a complex Banach space, $Z$ can be considered as a real Banach space and $f \rightarrow \operatorname{Re} f$ is an isometry from $Z_{\mathbb{C}}{ }^{*}$ onto $Z_{\mathbb{R}}{ }^{*}$. Thus, if $Y$ is a (complex) subspace and an $M$-ideal in $Z$, then $Y_{\mathbb{R}}$ is an $M$-ideal in $Z_{\mathbb{R}}$. Hence, proving proximinality of $M$-ideals in the case of real Banach spaces would also imply the result for the complex case (see Proposition 1.23 of [5]).

We start our proof by quoting a corollary of the Hahn-Banach theorem that is needed in the sequel. This corollary characterizes the set of nearest points from a subspace.

Lemma 1 ([7). Let $X$ be a Banach space, $Y$ a subspace of $X$ and $x$ an element of $X$. Then $y_{0}$ is in $P_{Y}(x)$ if and only if there exists an $f$ in $Y^{\perp}$ such that $\|f\|=1$ and $f\left(x-y_{0}\right)=\left\|x-y_{0}\right\|$.

We also need the following simple fact about $M$-ideals.

Remark 2. Let $Y$ and $W$ be subspaces of a Banach space $X$, with $Y$ contained in $W$. If $Y$ is an $M$-ideal in $X$, then $Y$ is an $M$-ideal in $W$.

To see this, let $Y$ be an $M$-ideal in $X$. If $f_{1}$ is in $W^{*}$, let $f$ be a Hahn-Banach extension of $f_{1}$ to $X$. Using (1), get $g$ in $Y^{\perp}$ and $h$ in $Z$ such that $f=g+h$ and $\|f\|=\|g\|+\|h\|$. Clearly, $f_{1}=f_{\mid W}=g_{\mid W}+h_{\mid W}$. We have $\left\|f_{1}\right\|=\left\|f_{\mid W}\right\|=\|f\|$ and by $(2),\|h\|=\left\|h_{\mid Y}\right\|=\left\|h_{\mid W}\right\|$. Now

$$
\|g\|=\left\|f_{\mid W}\right\|-\left\|h_{\mid W}\right\| \leq\left\|f_{\mid W}-h_{\mid W}\right\|=\left\|g_{\mid W}\right\| \leq\|g\| .
$$

Hence $\|g\|=\left\|g_{\mid W}\right\|$ and $\left\|f_{\mid W}\right\|=\left\|g_{\mid W}\right\|+\left\|h_{\mid W}\right\|$. It is now clear that $Y$ is an $M$-ideal in $W$ since the annihilator of $Y$, in $W^{*}$, is an $L$-summand of $W^{*}$.

Let $Y$ be an $M$-ideal in a Banach space $X$. Clearly $Y$ is proximinal in $X$ if and only if it is proximinal in every subspace $W$ of $X$, containing $Y$ as a hyperplane. By the above remark, $Y$ is an $M$-ideal in $W$. Thus, it suffices to prove proximinality of $Y$ in $X$, when $Y$ is a hyperplane in $X$.

Proposition 1. Let $X$ be a Banach space and let $Y$ be an $M$-ideal and a hyperplane in $X$. If there is a norm attaining functional $f$ in $X^{*}$ such that $\left\|f_{\mid Y}\right\|<\|f\|$, then $Y$ is proximinal in $X$. 
Proof. We assume the norm of $f$ is one and write $f=h+g$, with $h$ in $Y^{\perp}$ and $g$ in $Z$, where $Z$ is given by (2). Since

$$
\|g\|=\left\|f_{\mid Y}\right\|<\|f\|=1=\|h\|+\|g\|,
$$

$h$ is a non-zero element of $Y^{\perp}$.

Select any $x$ in $X \backslash Y$. Then there is a non-zero scalar $\alpha$ such that $f$ attains its norm at $\alpha x+y$, for some $y$ in $Y$. We have

$$
\begin{aligned}
\|\alpha x+y\| & =f(\alpha x+y)=h(\alpha x+y)+g(\alpha x+y) \\
& \leq\|h\|\|\alpha x+y\|+\|g\|\|\alpha x+y\| \\
& =\|\alpha x+y\| .
\end{aligned}
$$

It is now clear that $\frac{h}{\|h\|}(\alpha x+y)=\|\alpha x+y\|$ and consequently $-\frac{y}{\alpha}$ is in $P_{Y}(x)$ by Lemma 1. For a scalar $a$, and $y$ in $Y, P_{Y}(a x+y)=a P_{Y}(x)+y$, and so $Y$ is proximinal in $X$.

We now proceed to show that $f$ as in Proposition 1 can be constructed for any hyperplane of a Banach space.

Theorem 1. Let $X$ be Banach space and let $Y$ be a hyperplane in $X$. Then there is a norm attaining functional $f$ in $X^{*}$ such that $\left\|f_{\mid Y}\right\|<\|f\|$.

Proof. Choose a norm one element $x$ in $X \backslash Y$ and define $g$ in $X^{*}$ by $g(y+\alpha x)=\alpha$ for any scalar $\alpha$ and $y$ in $Y$. Then $\|g\| \geq 1$ and $g_{\mid Y}=0$. The Bishop-Phelps Theorem guarantees that there is a norm attaining functional $f$ in $X^{*}$ with $\|f-g\|<\frac{1}{3}$. Clearly $\left\|f_{\mid Y}\right\|<\frac{1}{3}$, while $\|f\|>\frac{2}{3}$.

The theorem below follows immediately from Proposition 1 and Theorem 1.

Theorem 2 (See Proposition 1.1 of [5]). Let $X$ be a Banach space and let $Y$ be an $M$-ideal in $X$. Then $Y$ is proximinal in $X$.

\section{ACKNOWLEDGEMENT}

The authors would like to thank the referee for comments leading to a concise presentation of the results. The second-named author's research was supported by the CSIR Research Fellowship, and she would like to thank the CSIR for their financial support.

\section{REFERENCES}

[1] E. M. Alfsen and E.G. Effros, Structure in real Banach spaces, Part I and II, Ann. of Math. 96 (1972) 98-173. MR0352946(50:5432)

[2] T. Ando, Closed range theorems for convex sets and linear liftings, Pacific J. Math. 44 (1973) 393-410. MR0328546 (48:6888)

[3] E. Behrends, M-Structure and the Banach-Stone theorem, Lecture Notes in Math. 736, Springer, Berlin, Heidelberg, New York, 1979. MR0547509 (81b:46002)

[4] M. Fabian, P. Habala, P. Hajek, V. M. Santalucia, J. Pelant and V. Zizler, Functional Analysis and Infinite-Dimensional Geometry, Springer, Berlin, Heidelberg, New York, 2001. MR1831176 (2002f:46001)

[5] P. Harmand, D. Werner and W. Werner, $M$-ideals in Banach spaces and Banach algebras, Lecture Notes in Math. 1574. Springer, Berlin, Heidelberg, New York, 1993. MR.1238713 (94k:46022)

[6] Ka-Sing Lau, On a sufficient condition for proximity, Trans. Amer. Math. Soc. 251 (1979) 343-356. MR0531983 (81b:46033) 
[7] I. Singer, Best Approximation in Normed Linear spaces by Elements of Linear Subspaces, Springer, Berlin, Heidelberg, New York, 1970. MR 0270044 (42:4937)

[8] D. Yost, Best approximation and intersections of balls in Banach spaces, Bull. Austral. Math. Soc. 20 (1979) 285-300. MR0557239 (80m:46019)

Department of Mathematics, Pondicherry University, Kalapet, Pondicherry-605014, INDIA

E-mail address: pdyindumath@gmail.com

Department of Mathematics, Pondicherry University, Kalapet, Pondicherry-605014, INDIA

E-mail address: s_lalithambigai@yahoo.co.in 